\title{
LES study of precipitation/condensation dependance on cumulus clouds dynamics
}

\author{
Yefim L. Kogan \\ NorthWest Research Associates, Inc. Redmond, WA 98052-5164, USA \\ Correspondence: Yefim L. Kogan (ykogan@nwra.com)
}

Received: 15 January 2021 - Revised: 23 April 2021 - Accepted: 23 April 2021 - Published: 19 May 2021

\begin{abstract}
Parameters affecting condensation/evaporation rates (CR/ER) in trade wind cumulus clouds were analyzed using LES model simulations. The model was initialized with data observed during the RICO field project, and simulated in a rather large $50.0 \times 50.0 \times 4 \mathrm{~km}^{3}$ domain. 2031 clouds were analyzed seeking relationships between CR/ER and thermo-dynamical cloud parameters. The condensation/evaporation rates were analyzed by stratifying the clouds by their size. The analyzed parameters included, among others, integral mass and buoyancy fluxes, as well as cloud and rain water and drop concentration.

The results revealed rather remarkable relationship between integral condensation/evaporation rate and integral upward mass flux. Identified relathionship may be useful for parameterization of subgrid latent heat in meso and large-scale models.
\end{abstract}

\section{Introduction}

The latent heat release plays an important role in predicting the thermodynamic structure of cumulus convection. Its accurate formulation is challenging in meso and large-scale models, not least because of sub-grid scale microphysical variability. The goal of our LES study is to investigate the phase transition process which is the source of latent heat release in convective clouds, and, specifically, its dependance on cloud thermo-dynamical variables. The LES model we use (SAMBM) employs the dynamical core of the System for Atmospheric Modeling (SAM, Khairoutdinov and Randall, 2003) and the Bulk Microphysics tuned for shallow $\mathrm{Cu}$ convection (BM, Kogan, 2013). The observations from the RICO field campaign (vanZanten et al., 2011) were used for initializing the LES simulations conducted in a rather large $50.0 \times 50.0 \times 4 \mathrm{~km}^{3}$ domain $(500 \times 500 \times 100$ grid points $)$.

Over the course of the $32 \mathrm{~h}$ run, the simulation data was saved every $30 \mathrm{~min}$; from this archive a total of 2031 clouds were selected for analysis. Our initial attempt of using the "brute force" statistical approach to relate phase transition rates to the dynamical parameters did not succeed because of the complexity of the cloud system, consisting of clouds at various stages of their development. A better approach proved to be separation of the entire dataset into subsets stratified by the cloud size, maturity, parameters of precipitation.

Specifically, we employed the method used previously for PDF parameterization development (Kogan and Mechem, 2014, 2016); namely, the dataset was sorted by cloud top height and divided into four groups G1-G4, each of which condenses approximately equal amount of water vapor per second. The groups G1-G2 represented small clouds (1346 in G1 and 483 in G2). mostly at the growing stage, while groups G3-G4 (137 clouds in G3 and 65 in G4) contained mature or decaying clouds.

\section{Results}

Figure 1 shows mean and standard deviation of selected physical and precipitation cloud parameters in each group. G1 clouds are most numerous; they are also the smallest with cloud tops varying in the range from 1.34 to $2.3 \mathrm{~km}$. Their mean projected surface area is on average less than $2 \mathrm{~km}^{2}$ and mean volume is less than $1 \mathrm{~km}^{3}$. The linear increase by only $300 \mathrm{~m}$ in cloud height from G2 to G3, and further to G4 is accompanied by an exponential increase in cloud area and volume (Fig. 1a), as well as cloud and rain water (not shown). Ratio of rain to cloud water growth seems to be lag- 

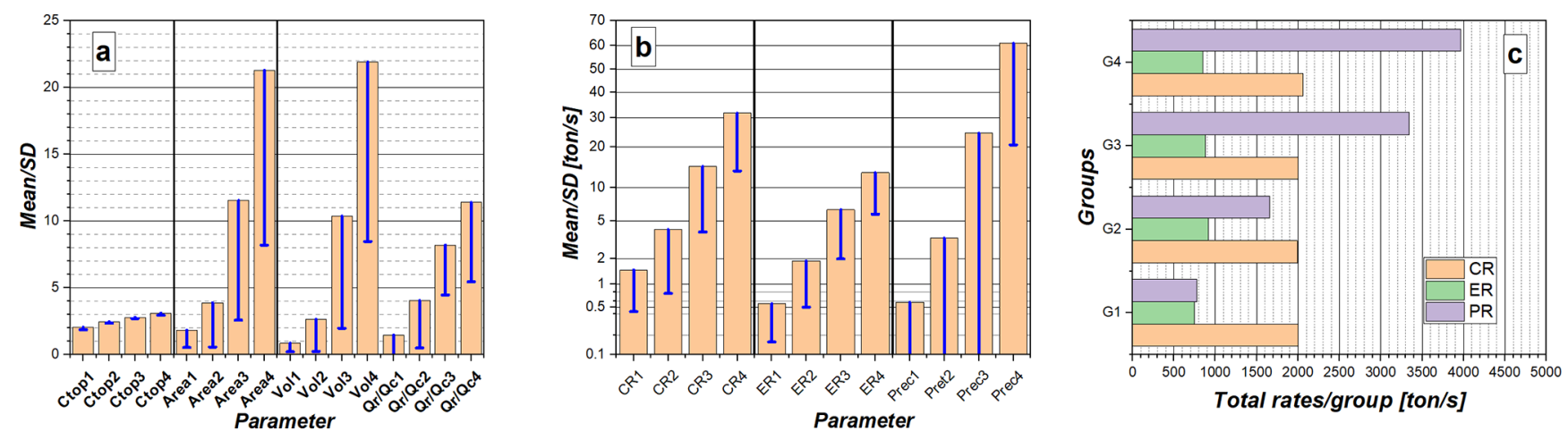

Figure 1. Mean and standard deviation of cloud physical parameters in each of the four groups. (a) cloud top, surface area, volume (Ctop, Area, Vol - in km, $\mathrm{km}^{2}, \mathrm{~km}^{3}$, respectively), and rain to cloud water ratio (in percentages divided by a factor of 10); (b) per cloud condensation/evaporation and precipitation rate (PR - integrated over the cloud surface area) in t/s; (c) total CR/ER/PR integrated over the group $(\mathrm{t} / \mathrm{s})$.

ging behind the growth of cloud volume, e.g., nearly ninefold increase in cloud volume from G2 to G4 results in less than three-fold increase in QR4/QC4. This does not imply, however, that larger clouds condense less effectively; it simply reflects the acceleration in rain formation as clouds grow larger.

Mean condensation/evaporation rates (CR/ER) shown in Fig. $1 \mathrm{~b}$ are more in line with the increase in volume (note the probability scale on the $y$-axis). So, about nine-fold increase in Vol4/Vol2 ratio corresponds to about eight time increase in CR4/CR2, and about seven times increase in ER4/ER2. Obviously, the majority of clouds in groups G1 and G2 are at a growing stage, as their condensation rates are larger than the precipitation rates (PR), while the opposite is true for larger clouds in G3-G4 (Fig. 1b).

Even larger, exponential increase is seen when analyzing PR. For clouds in G1 PRs are very small, only $0.6 \mathrm{~mm} / \mathrm{h}$. Clouds in G2 are three time larger in volume, but their PRs are six times larger. The clouds in G3 have about four times larger volumes than G2 clouds, but their PRs increase more than seven times. Even more dramatic difference exists for G4 clouds, where nine times increase in volume leads to 18 times larger precipitation rates.

While Fig. 1a-b show mean cloud parameters in each group, the Fig. 1c shows total contribution of these parameters, i.e. integrated over the whole group. Each group, by design, contributes approximately equal amount of condensation per second. The fraction of evaporation to condensation rates $(\mathrm{ER} / \mathrm{CR})$ is on average about $40 \%-45 \%$. This fraction is smaller for G1 and G4 clouds ( $37.4 \%$ and $41.4 \%$ ), while larger for G2-G3 clouds ( $45.9 \%$ and $44.0 \%$ ). Evidently, G1 clouds are predominantly growing, therefore evaporation lags behind condensation, while G2-G3 clouds are at a mature stage, and have already well formed quasi-stable dynamical updraft/downdraft structure where condensation and evaporation ls balanced. Larger rain water content in G4 clouds contributes more to precipitation and somewhat less to evaporation, which may explain the reduced fraction of evaporation in G4 (41.4\%) compared to G3 clouds.

Together G4 and G3 account for three quarter of total precipitation; G1 and G2 contribute, respectively $8 \%$ and $17 \%$. As already mentioned, these groups precipitate less than condense, i.e., they are still growing, while G3, and especially G4 clouds precipitate about $70 \%$ and $100 \%$ more than condense, that is, they are losing water and, therefore, at the stage of decay.

Analysis of correlation between CR/ER and the thermodynamical parameters which may affect CR/ER was conducted for clouds in each group separately. The analyzed parameters were integrated over the whole cloud volume. These include: up and down mass flux MF (defined as air density $\rho$ times vertical velocity: $\rho W)$, cloud and rain water content (QC and $\mathrm{QR}$ ), up and down buoyancy flux BF (defined as $c_{\mathrm{p}} \rho \theta^{\prime} W^{\prime}$. where $c_{\mathrm{p}}$ is the specific heat of air and $\theta^{\prime}$ is the virtual temperature perturbation). As the variables were integrated over the whole cloud, and the size of the cloud volume is on the order of billions of cubic meters, it is convenient to normalize the variables by a unit volume $V_{0}=10^{9} \mathrm{~m}^{3}\left(\equiv 1 \mathrm{~km}^{3}\right)$.

Figure 2 shows, as an example, results of the correlation analysis for clouds in G2; the results for clouds in other groups are similar. One can note exceptionally high correlation between condensation rate and upward (Plus) mass flux (MFP). Correlation between CR and upward buoyancy flux (BFP) is also high, but weaker than with MFP. Similar strong correlation exists with cloud water QC. As one might expect, the correlation between condensation and downward (Minus) fluxes (MFM, BFM), as well as rain parameters (QR) is weaker. For other cloud groups results are similar with the same conclusion: the strongest correlation is between CR and MFP.

The evaporation rate (ER) (Fig. 3) also has stronger correlation with the upward (MFP) than with downward mass flux (MFM) (Fig. 3a-b). Buoyancy flux determines ER worse than upward mass flux (Fig. 3c-d), however, its downward 

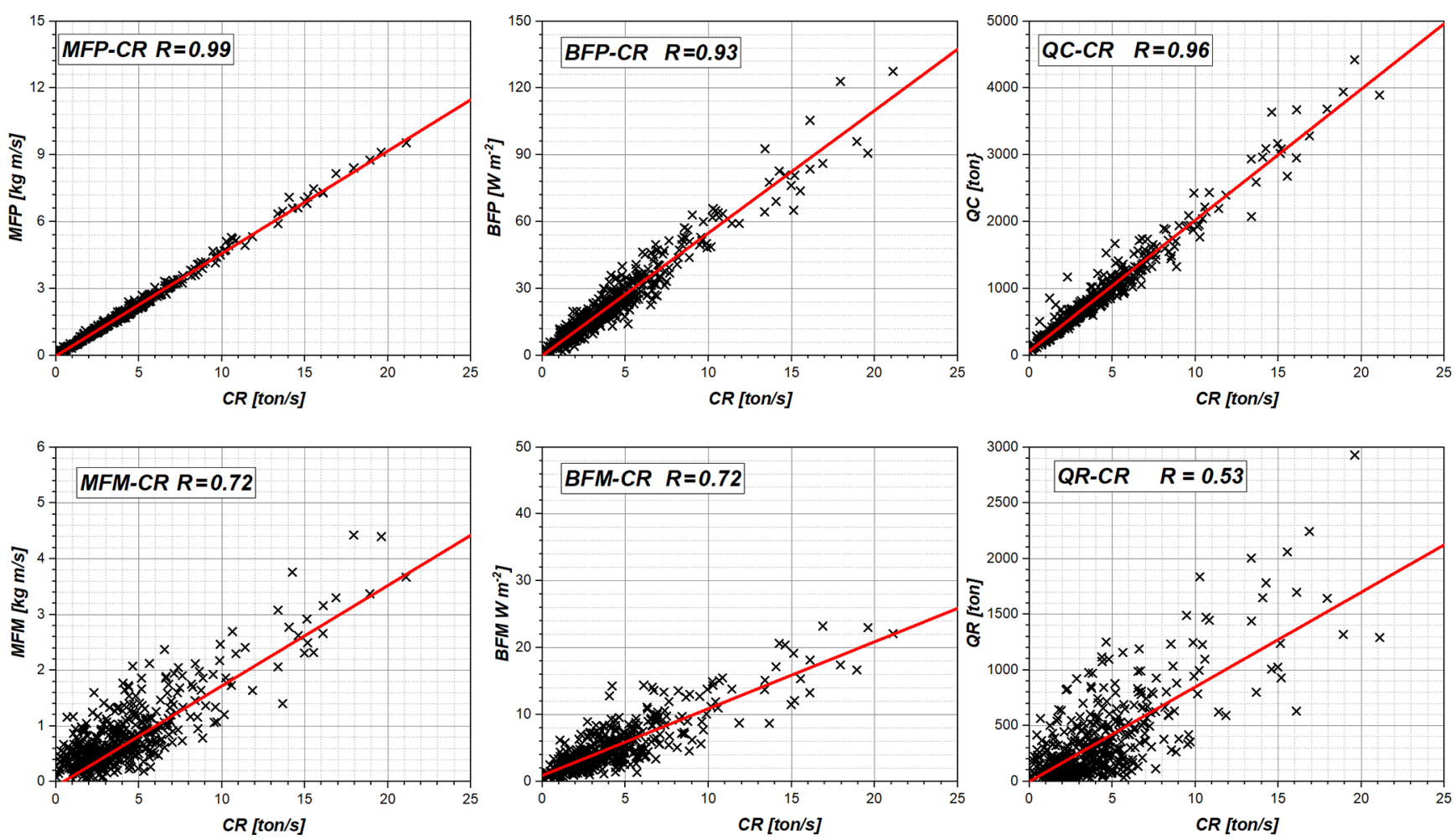

Figure 2. Correlation of condensation rate (CR) with parameters denoted in plot legends. Group G2 $R$ is the correlation coefficient.
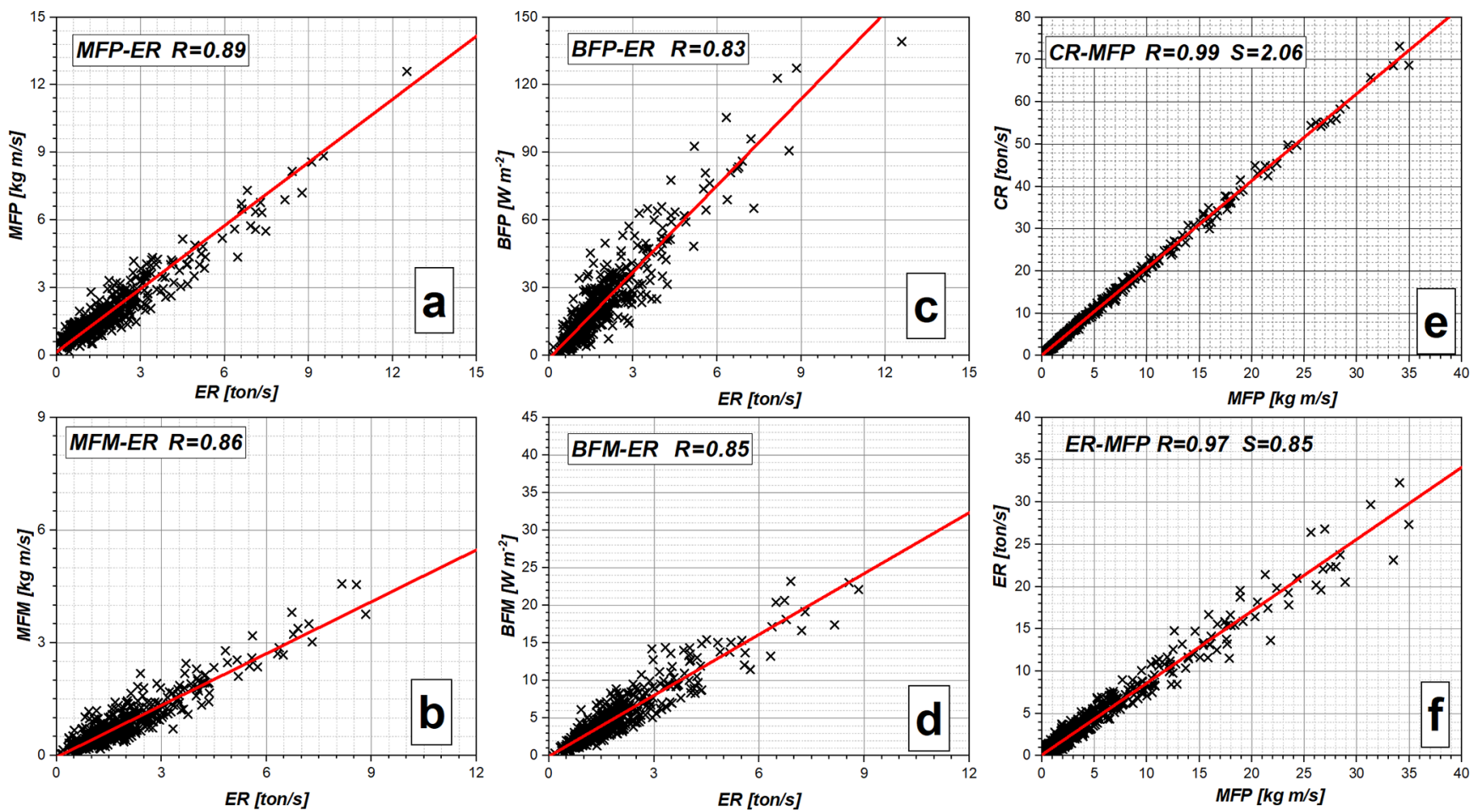

Figure 3. Correlation plots of: (a-d) ER with mass and buoyancy flux for G2 clouds, (e-f) - CR/ER with upward mass flux MFP for clouds from all groups combined. $R$ is the correlation coefficient, $\mathrm{S}$ is the slope of the linear fit shown by the red line. 
component (BFM) correlates with ER slightly stronger than its upward component BFP. All in all, it is rather remarkable that the upward mass flux is the parameter which defines both condensation and evaporation in a cloud.

What is also remarkable, is that the slope of the linear fit approximating the correlation between CR/ER and MFP only slightly depends on cloud group, i.e., on cloud top height. The scatter plots in Fig. 3e-f illustrate this fact which can be expressed as a linear relationship between phase transition rate (PTR) and upward mass flux:

$\mathrm{PTR}=\alpha \mathrm{MFP}$

where $\alpha=2.06$ for condensation $(\mathrm{PTR} \equiv \mathrm{CR}$ ) and $\alpha=-0.85$ for evaporation (PTR $\equiv \mathrm{ER}$ ).

\section{Conclusion}

Based on the LES model data, we analyzed condensation/evaporation parameters, and their correlation with thermodynamical parameters of trade-wind cumulus convective clouds. A very strong correlation was found not only between the condensation, but also evaporation rate and upward mass flux (all parameters were integrated over the whole cloud volume). While good correlation between the upward mass flux and condensation is not surprising (obviously due to larger supersaturation in stronger updrafts), the very high correlation coefficient $(R=0.99)$, as well as the role of the upward mass flux in determining the evaporation rate, is remarkable. Whether this finding will hold for vertically dependent variables needs to be further investigated.

Code availability. The software code for data analysis was developed by the author and is available upon request.

Data availability. Analysis data is available upon request from the author.

Competing interests. The author declares that there is no conflict of interest.
Special issue statement. This article is part of the special issue "Applied Meteorology and Climatology Proceedings 2020: contributions in the pandemic year".

Acknowledgements. This investigation was supported by ONR Grant N00014-20-1-2050. The author is grateful to Vaughan Phillips and anonymous reviewer for constructive comments. The computing for this project was performed at the OU Supercomputing Center for Education and Research (OSCER) at the University of Oklahoma.

Financial support. This research has been supported by the Office of Naval Research (grant no. N00014-20-1-2050).

Review statement. This paper was edited by Emily Gleeson and reviewed by Vaughan Phillips and one anonymous referee.

\section{References}

Khairoutdinov, M. F. and Randall, D. A.: Cloud resolving modeling of the ARM summer 1997 IOP: Model formulation, results, uncertainties, and sensitivities, J. Atmos. Sci., 60, 607-625, 2003.

Kogan, Y. L.: A Cumulus Cloud Microphysics Parameterization for Cloud-Resolving Models, J. Atmos. Sci., 70, 1423-1436, 2013.

Kogan, Y. L. and Mechem, D. B.: A PDF based microphysics parameterization for shallow cumulus clouds, J. Atmos. Sci., 71, 1070-1089, 2014.

Kogan, Y. L. and Mechem, D. B.: A PDF-Based Formulation of Microphysical Variability in Cumulus Congestus Clouds, J. Atmos. Sci., 73, 167-184, 2016.

VanZanten, M. C., Stevens, B., Nuijens, L., Siebesma, A. P., Ackerman, A. S., Burnet, F., Cheng, A., Couvreux, F., Jiang, H., Khairoutdinov, M., Kogan, Y., Lewellen, D. C., Mechem, D., Nakamura, K., Noda, A., Shipway, B. J., Slawinska, J., Wang, S., and Wyszogrodzki, A.: Controls on precipitation and cloudiness in simulations of trade-wind cumulus as observed during RICO, J. Adv. Model. Earth Syst., 3, M06001, https://doi.org/10.1029/2011MS000056, 2011. 